\title{
Matrix polynomials with partially prescribed eigenstructure: eigenvalue sensitivity and condition estimation
}

\author{
FERMIN S. VILOCHE BAZÁN* \\ Department of Mathematics, Federal University of Santa Catarina \\ Florianópolis, Santa Catarina, 88040-900, Brazil \\ CERFACS Technical Report TR/PA/04/64 \\ E-mail: fermin@mtm.ufsc.br
}

\begin{abstract}
Let $P_{m}(z)$ be a matrix polynomial of degree $m$ whose coefficients $A_{t} \in \mathbb{C}^{q \times q}$ satisfy a recurrence relation of the form: $h_{k} A_{0}+h_{k+1} A_{1}+\cdots+h_{k+m-1} A_{m-1}=h_{k+m}$, $k \geq 0$, where $h_{k}=R Z^{k} L \in \mathbb{C}^{p \times q}, R \in \mathbb{C}^{p \times n}, Z=\operatorname{diag}\left(z_{1}, \ldots, z_{n}\right)$ with $z_{i} \neq z_{j}$ for $i \neq j$, $0<\left|z_{j}\right| \leq 1$, and $L \in \mathbb{C}^{n \times q}$. The coefficients are not uniquely determined from the recurrence relation but the polynomials are always guaranteed to have $n$ fixed eigenpairs, $\left\{z_{j}, l_{j}\right\}$, where $l_{j}$ is the $j$ th column of $L^{*}$. In this paper, we show that the $z_{j}$ 's are also the $n$ eigenvalues of an $n \times n$ matrix $C_{A}$; based on this result the sensitivity of the $z_{j}$ 's is investigated and bounds for their condition numbers are provided. The main result is that the $z_{j}$ 's become relatively insensitive to perturbations in $C_{A}$ provided that the polynomial degree is large enough, the number $n$ is small, and the eigenvalues are close to the unit circle but not extremely close to each other. Numerical results corresponding to a matrix polynomial arising from an application in system theory show that low sensitivity is possible even if the spectrum presents clustered eigenvalues.
\end{abstract}

Mathematical subject classification: $65 \mathrm{~F} 20,65 \mathrm{~F} 15$.

Key words: matrix polynomials, block companion matrices, departure from normality, eigenvalue sensitivity, controllability Gramians.

\#616/04. Received: 09/IX/04. Accepted: 12/XII/04.

*This research was performed while the author was a visitor at CERFACS, Toulouse, France, and was sponsored by CNPq, Brazil, grant 201407/03-5(NV). 


\section{Introduction}

We are concerned with matrix polynomials

$$
P_{m}(z)=A_{0}+z A_{1}+\cdots+z^{m-1} A_{m-1}-z^{m} I, \quad z \in \mathbb{C},
$$

whose coefficients $A_{t} \in \mathbb{C}^{q \times q}(t=0: m-1)$ satisfy a recurrence relation of the form

$$
h_{k} A_{0}+h_{k+1} A_{1}+\cdots+h_{k+m-1} A_{m-1}=h_{k+m}, \quad k=0,1, \cdots
$$

where $h_{k} \in \mathbb{C}^{p \times q}$. The coefficients, known as predictor parameters, reflect intrinsic properties of the sequence $\left\{h_{k}\right\}$ such as frequencies, damping factors, plane waves, etc, whose estimation from a finite data set $\left\{h_{k}\right\}_{k=0}^{T}$, is an important problem in science and engineering [1, 19, 20, 21, 22, 28]. In this work, we concentrate on polynomials arising in applications where the data are assumed to be modeled as

$$
h_{k}=R Z^{k} L, \quad k=0,1, \ldots
$$

where $Z=\operatorname{diag}\left(z_{1}, \ldots, z_{n}\right)$ with $z_{i} \neq z_{j}$ for $i \neq j,\left|z_{i}\right| \leq 1, R \in \mathbb{C}^{p \times n}$ is of rank $p$ and $L \in \mathbb{C}^{n \times q}$ of rank $q$ with rows scaled to unit length. Also, as usual in the applications of interest, we shall assume that $n$ is a small number.

Model (3) covers, e.g., impulse response samples of dynamic linear systems $[1,4,19,28,29]$, where the $z$ 's are system poles, time domain nuclear magnetic resonance (NMR) data [26, 27], and time series defined by

$$
h_{k}=\sum_{j=1}^{d} f_{j} \cos k \lambda_{j}+g_{j} \sin k \lambda_{j},
$$

where $f_{j}, g_{j} \in \mathbb{C}^{p \times 1}$, the $z$ 's are of the form $z_{j}=e^{i \lambda_{j}}(l=\sqrt{-1}), n=2 d$, and $q=1$ (see [22] and references therein).

In these applications, one wants to estimate the parameters $z_{j}$ and the matrices $R, L$ from a finite data set $\left\{h_{k}\right\}_{k=0}^{T}$. The problem is difficult as $n$ is not always known in advance and the available data are corrupted by noise. However, a relatively simple polynomial-based approach can be used. The approach relies on the fact that if the data are free of noise and the coefficients are estimated from a linear system constructed by stacking $m^{\prime}$ successive recurrence relations, 
where we assume that $m^{\prime} \geq m \geq n$, and $n$ is the rank of the coefficient matrix, then $P_{m}(z)$ has $z_{j}(j=1: n)$ as eigenvalue and $l_{j}(j=1: n)$, the $j$ th column of $L^{*}$, as associated left eigenvector [1, 19,28] (the star symbol denotes conjugate transpose). Details about eigenvalues of matrix polynomials can be found in [12]. The remaining $m q-n$ eigenvalues have no physical meaning and are commonly known as spurious eigenvalues. Once the eigenpairs $\left\{z_{j}, l_{j}\right\}$ are available, the estimation of $R$ is straightforward. The same approach can be used in the noisy data case but some criterion is needed to separate the eigenvalues of interest from the spurious ones.

Note that since $\left\{z_{j}, l_{j}\right\}$ are eigenpairs of $P_{m}(z)$, then there holds

$$
l_{j}^{*} P_{m}\left(z_{j}\right)=0, \quad j=1: n .
$$

This is an underdetermined linear system of the form

$$
\mathcal{K}_{m} X_{A}=Z^{m} L
$$

where $X_{A}^{T}=\left[\begin{array}{lll}A_{0}^{T} & \cdots & A_{m-1}^{T}\end{array}\right]$ and $\mathcal{K}_{m}$ is an $n \times m q$ full-rank Krylov matrix defined by

$$
\mathcal{K}_{m}=\left[\begin{array}{lllll}
L & Z L & Z^{2} L & \cdots & Z^{m-1} L
\end{array}\right] .
$$

Thus, all polynomials whose coefficients satisfy (2) (and hence (5)) will have $n$ fixed eigenpairs, $\left\{z_{j}, l_{j}\right\}$, but the remainder of their eigenstructure will depend on the solution chosen. In the sequel we refer to the $z_{j}$ 's as prescribed eigenvalues of $P_{m}(z)$ and to the polynomial itself as a polynomial with partially prescribed eigenstructure, or shortly, as a predictor matrix polynomial. For applications involving predictor polynomials, the reader is referred to [1, 19, 21, 25, 29, 22].

We observe also that associated with $P_{m}(z)$ there is a block companion matrix $C_{A}$ defined by

$$
C_{A}=\left[\begin{array}{ccccc}
0 & 0 & \cdots & 0 & A_{0} \\
I_{q} & 0 & \cdots & 0 & A_{1} \\
0 & I_{q} & \cdots & 0 & A_{2} \\
\vdots & \vdots & \ddots & \vdots & \vdots \\
0 & 0 & \cdots & I_{q} & A_{m-1}
\end{array}\right]
$$

This matrix has the same eigenvalues as $P_{m}(z)$ [12], left eigenvectors of the form $\ell^{*}=\left[l^{*} z l^{*} \cdots z^{m-1} l^{*}\right]$ with $l$ a left eigenvector of $P_{m}(z)$, and satisfies the 
matrix equation

$$
\mathcal{K}_{m} C_{A}=Z \mathcal{K}_{m}
$$

In practice the coefficients $A_{t}$ are never known exactly and one has to analyze the sensitivity of the $z_{j}$ 's to perturbations in $A_{t}$. The problem has received the attention of many researchers and many sensitivity analyses for the scalar case (i.e., for $q=1$ ) are now available, see, e.g., [2, 6, 17, 21, 25]. Some results concerning sensitivity of eigenvalues of general matrix polynomials can be found in $[14,24]$. However, to the best of our knowledge nothing has been done on sensitivity analysis of prescribed eigenvalues of predictor polynomials for $q>1$. The goal of this work is to carry out a sensitivity analysis of prescribed eigenvalues only, focusing on the influence of the polynomial degree on such sensitivity. We show that this can be done by relating the $z_{j}$ 's to a small $n \times n$ matrix obtained by projecting $C_{A}$ onto an appropriate subspace and then analyzing the projected eigenproblem. As a result, simple estimates of measures of sensitivity of the $z_{j}$ 's in the form of informative upper bounds are given.

The following notation is used throughout the paper. For $A \in \mathbb{C}^{m \times n},\|A\|_{2}$ and $\|A\|_{F}$ denote the 2-norm (or spectral) and Frobenius norm of $A$, respectively. $A^{\dagger}$ denotes the Moore-Penrose pseudo-inverse of $A$. The $i$ th singular value of $A$ is denoted by $\sigma_{i}(A)$. The 2-norm condition number of $A, \kappa(A)$, is defined by $\kappa(A)=\|A\|_{2}\left\|A^{\dagger}\right\|_{2}$. The spectrum of $A \in \mathbb{C}^{n \times n}$ is denoted by $\lambda(A)$. The identity matrix of order $n$ is denoted by $I_{n}$ and its $j$ th column by $e_{j}$.

The paper is organized as follows. In Section 2, we describe results concerning the singular values of projected companion matrices by extending the work in [5]. The results obtained are then exploited in Section 3, in which we analyze the departure of the projected companion matrix from normality. In Section 4, we analyze the condition numbers of the $z_{j}$ 's introduced by Wilkinson [30], and the overall 2-norm condition number of the related eigenvalue problem. We show that these measures of sensitivity are governed by the 2-norm condition number of the Krylov matrix and conclude that eigenvalues near the unit circle become relatively insensitive to noise provided that the polynomial degree is large enough and the eigenvalues themselves are not extremely close to each other. In addition to this, we provide estimates for the 2-norm condition number of controllability Gramians of multi-input multi-output discrete dynamical sys- 
tems in diagonal form. Numerical results corresponding to a matrix polynomial arising from an application in system theory show that low sensitivity is possible even if some eigenvalues are clustered.

\section{Singular value analysis of the projected companion matrix}

In order to start our analysis we introduce a new block companion matrix associated with the prescribed eigenvalues. Let $C_{B}$ be defined by

$$
C_{B}=\left[\begin{array}{ccccc}
B_{0} & I_{q} & 0 & \cdots & 0 \\
B_{1} & 0 & I_{q} & \cdots & 0 \\
\vdots & \vdots & \ddots & \ddots & \vdots \\
B_{m-2} & 0 & \cdots & 0 & I_{q} \\
B_{m-1} & 0 & \cdots & 0 & 0
\end{array}\right]
$$

whose first column block, denoted by $X_{B}$, is any solution of the underdetermined linear system $\mathcal{K}_{m} X_{B}=Z^{-1} L$. This definition ensures that $z_{j}^{-1}(j=1: n)$ is an eigenvalue of $C_{B}$ and that there holds

$$
\mathcal{K}_{m} C_{B}=Z^{-1} \mathcal{K}_{m}
$$

Let the columns of $\mathcal{V}$ form an orthonormal basis for $\mathcal{R}\left(\mathcal{K}_{m}^{*}\right)$, the column space of $\mathcal{K}_{m}^{*}$. Notice that because of (8) and (10), $\mathcal{R}\left(\mathcal{K}_{m}^{*}\right)$ is a left invariant subspace of both $C_{A}$ and $C_{B}$ associated with the eigenvalues of interest. Let $C_{A}(m, q)$ and $C_{B}(m, q)$ be the matrices obtained by projecting $C_{A}$ and $C_{B}$ onto $\mathcal{R}\left(\mathcal{K}_{m}^{*}\right)$, that is,

$$
C_{A}(m, q)=\mathcal{V}^{*} C_{A} \mathcal{V}, \quad C_{B}(m, q)=\mathcal{V}^{*} C_{B} \mathcal{V} .
$$

Then it is clear that

$$
\lambda\left(C_{A}(m, q)\right)=\left\{z_{1}, \ldots z_{n}\right\}, \quad \lambda\left(C_{B}(m, q)\right)=\left\{z_{1}^{-1}, \ldots z_{n}^{-1}\right\} .
$$

The goal of this section is to analyze the singular values of $C_{A}(m, q)$, focusing on their behavior as function of $m, q$. Before proceeding we observe that when the dependence of $C_{A}(m, q)$ and $C_{B}(m, q)$ on $m, q$ is not important for the 
understanding, these matrices will be denoted by $C_{A}$ and $C_{B}$. Notice also that the projector orthogonal onto $\mathcal{R}\left(\mathcal{K}_{m}^{*}\right)$, denoted by $\mathcal{P}$, satisfies

$$
\mathcal{P}=\mathcal{V} \mathcal{V}^{*}=\mathcal{K}_{m}^{\dagger} \mathcal{K}_{m}
$$

Two lemmas are needed.

Lemma 2.1. For $m \geq n$ and $q \geq 1$ there holds $C_{A}=C_{B}^{-1}$.

Proof. Since $\mathcal{K}_{m} \mathcal{K}_{m}^{*}$ is positive definite Hermitian, it is clear that the columns of $\mathcal{V}=\mathcal{K}_{m}^{*}\left(\mathcal{K}_{m} \mathcal{K}_{m}^{*}\right)^{-1 / 2}$ form an orthonormal basis for $\mathcal{R}\left(\mathcal{K}_{m}^{*}\right)$. Using this basis and the definitions of $C_{A}$ and $C_{B}$ we have

$$
C_{A} C_{B}=\left(\mathcal{K}_{m} \mathcal{K}_{m}^{*}\right)^{-1 / 2} \mathcal{K}_{m} C_{A} \mathcal{K}_{m}^{*}\left(\mathcal{K}_{m} \mathcal{K}_{m}^{*}\right)^{-1 / 2}\left(\mathcal{K}_{m} \mathcal{K}_{m}^{*}\right)^{-1 / 2} \mathcal{K}_{m} C_{B} \mathcal{K}_{m}^{*}\left(\mathcal{K}_{m} \mathcal{K}_{m}^{*}\right)^{-1 / 2}
$$

This reduces to identity on using (8), (10), and the fact that $\mathcal{K}_{m} \mathcal{K}_{m}^{\dagger}=I$.

Lemma 2.2. Let $A=A_{1} A_{1}^{*}-B_{1} B_{1}^{*}$ with $A_{1} \in \mathbb{C}^{n \times p}$ and $B_{1} \in \mathbb{C}^{n \times q}$. Assume $\operatorname{rank}\left(\left[\begin{array}{ll}A_{1} & B_{1}\end{array}\right]\right)=p+q<n$. Then, the number of positive, negative, and zero eigenvalues of $A$, is $p, q$, and $n-(p+q)$, respectively.

Proof. Let the nonzero eigenvalues of $A$ be arranged so that $\lambda_{1}(A) \geq$ $\lambda_{2}(A) \geq \cdots \geq \lambda_{p+q}(A)$. Our proof relies on the minimax principle for eigenvalues [11]:

$$
\lambda_{k}(A)=\max _{\operatorname{dim}(S)=k} \min _{x \in S, x \neq 0} \frac{x^{*} A x}{x^{*} x} .
$$

Let the matrix $P=\left[B_{1} \mid A_{1}\right]$ have a $\mathrm{QR}$ factorization

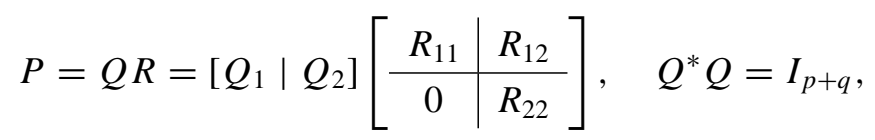

where $Q, R$ are partitioned such that $Q_{1} \in \mathbb{C}^{n \times q}, Q_{2} \in \mathbb{C}^{n \times p}, R_{11} \in \mathbb{C}^{q \times q}$, $R_{12} \in \mathbb{C}^{p \times p}$ and $R_{22} \in \mathbb{C}^{p \times p}$. Clearly, both $R_{11}$ and $R_{22}$ are nonsingular. From (13) it follows that

$$
B_{1}=Q_{1} R_{11}, \quad \text { and } \quad A_{1}=Q_{1} R_{12}+Q_{2} R_{22} .
$$


Substituting $B_{1}$ and $A_{1}$ into $A$, it follows that the projection of $A$ onto $\mathcal{R}\left(Q_{2}\right)$, the subspace spanned by the columns of $Q_{2}$, is

$$
Q_{2}^{*} A Q_{2}=R_{22} R_{22}^{*} .
$$

Let $x \in \mathcal{R}\left(Q_{2}\right), x \neq 0$. Then, because $Q_{2}^{*} A Q_{2}$ is positive definite by (14), putting $x=Q_{2} \beta \in \mathbb{C}^{p}, \beta \neq 0$, we have

$$
\min _{x \in \mathcal{R}\left(Q_{2}\right), x \neq 0} \frac{x^{*} A x}{x^{*} x} \geq \min _{x \in \mathcal{R}\left(Q_{2}\right), x \neq 0} \frac{\beta^{*} Q_{2}^{*} A Q_{2} \beta}{\beta^{*} \beta}>0,
$$

and so, by the minimax principle, we conclude that $A$ has at least $p$ positive eigenvalues. Considering matrix $-A$ instead of $A$ and proceeding as before it follows that $A$ has at least $q$ negative eigenvalues. Apart from this, it is clear that $A$ has $n-(p+q)$ zero eigenvalues. From these conclusions the assertions of the lemma follow.

In order to describe our results concerning the singular values of $C_{m, q}$, we first notice that the Krylov matrix $\mathcal{K}_{m}$ becomes a weighted Vandermonde matrix when $q=1$. When the weights are all ones this matrix will be denoted by $W_{m}$. Let the columns of $\breve{V}$ form an orthonormal basis for $\mathcal{R}\left(W_{m}^{*}\right)$. Then the orthogonal projector onto $\mathcal{R}\left(W_{m}^{*}\right), \breve{\mathcal{P}}$, satisfies

$$
\breve{\mathcal{P}}=W_{m}^{\dagger} W_{m}=\breve{\mathcal{V}} \breve{V}^{*}
$$

Using this notation we set

$$
p_{1}=W_{m}^{\dagger} e, \quad x^{+}=W_{m}^{\dagger} Z^{m} e,
$$

where $e=[1, \cdots, 1]^{T} \in \mathbb{R}^{n}$.

We are now ready to describe the singular spectrum of matrix $C_{A}(m, q)$.

Theorem 2.3. Let the singular values of $C_{A}(m, q)$ be arranged so that $\sigma_{1}\left(C_{A}\right) \geq \cdots \geq \sigma_{n}\left(C_{A}\right)$. Assume that $\operatorname{rank}\left(\left[\begin{array}{ll}Z^{m} L & L\end{array}\right]\right)=2 q$. Then, for $1 \leq q<n / 2$, there holds

$$
\begin{array}{ll}
\sigma_{i}\left(C_{A}\right)>1 & i=1: q, \\
\sigma_{i}\left(C_{A}\right)=1, & i=q+1: n-q, \\
0<\sigma_{i}\left(C_{A}\right)<1 & i=n-q+1: n .
\end{array}
$$


Furthermore, if $q=1$ the singular values of $C_{A}(m, 1)$ do not depend on the matrix $L$ defined in (3), but rather on the Vandermonde matrix $W_{m}$. In this case they are given by

$$
\begin{aligned}
& \sigma_{1}^{2}\left(C_{A}(m, 1)\right)=\frac{1}{2}\left[2+\left\|x^{+}\right\|_{2}^{2}-\left\|p_{1}\right\|_{2}^{2}+\sqrt{\left(\left\|x^{+}\right\|_{2}^{2}+\left\|p_{1}\right\|_{2}^{2}\right)^{2}-4\left|x_{0}\right|^{2}}\right] \\
& \sigma_{j}^{2}\left(C_{A}(m, 1)\right)=1, j=2: n-1 . \\
& \sigma_{n}^{2}\left(C_{A}(m, 1)\right)=\frac{1}{2}\left[2+\left\|x^{+}\right\|_{2}^{2}-\left\|p_{1}\right\|_{2}^{2}-\sqrt{\left(\left\|x^{+}\right\|_{2}^{2}+\left\|p_{1}\right\|_{2}^{2}\right)^{2}-4\left|x_{0}\right|^{2}}\right]
\end{aligned}
$$

where $x_{0}$ denotes the first component of $x^{+}$.

Proof. We use the fact that the squared singular values of $C_{A}$ are eigenvalues of $C_{A} C_{A}^{*}$. In fact, using the definition of $C_{A}$,

$$
C_{A} C_{A}^{*}=\mathcal{V}^{*} C_{A} \mathcal{V} \mathcal{V}^{*} C_{A}^{*} \mathcal{V}=\mathcal{V}^{*} C_{A} \mathcal{P} C_{A}^{*} \mathcal{V}=\mathcal{V}^{*} C_{A} C_{A}^{*} \mathcal{V} .
$$

The last equality comes from the fact that $\mathcal{P} C_{A}^{*} \mathcal{V}=C_{A}^{*} \mathcal{V}$ because $\mathcal{V}$ is a basis of the right invariant subspace of $C_{A}^{*}$ associated with prescribed eigenvalues. Now notice that if we write $C_{A}=\left[E_{2} E_{2} \cdots E_{m} X_{A}\right]$, where $E_{j}$ denotes the block column vector having its $j$ th entry equal to $I_{q}$ and the remaining ones equal to the zero matrix, then

$$
C_{A} C_{A}^{*}=E_{2} E_{2}^{*}+\cdots+E_{m} E_{m}^{*}+X_{A} X_{A}^{*},
$$

and this can be rewritten as

$$
C_{A} C_{A}^{*}=I_{m q}-E_{1} E_{1}^{*}+X_{A} X_{A}^{*} .
$$

Hence, using the fact that $X_{A}$ solves the system (5), which implies that $X_{A}=X_{A}^{+}+N$, where $N$ is a matrix whose columns belong to $\mathcal{N}\left(\mathcal{K}_{m}\right)=$ $\left[\mathcal{R}\left(\mathcal{K}_{m}^{*}\right)\right]^{\perp}$, we have

$$
\begin{aligned}
C_{A} C_{A}^{*} & =I_{n}-\mathcal{V}^{*} E_{1} E_{1}^{*} \mathcal{V}+\mathcal{V}^{*} X_{A}^{+} X_{A}^{+*} \mathcal{V} \\
& =I_{n}-\mathcal{V}^{*} P_{1} P_{1}^{*} \mathcal{V}+\mathcal{V}^{*} X_{A}^{+} X_{A}^{+*} \mathcal{V},
\end{aligned}
$$

where

$$
P_{1}=\mathcal{K}_{m}^{\dagger} L=\mathcal{P} E_{1}, \quad X_{A}^{+}=\mathcal{K}_{m}^{\dagger} Z^{m} L
$$





nonsingular. From this and the assumption that $\operatorname{rank}\left(\left[Z^{m} L L\right]\right)=2 q$ it follows that $\operatorname{rank}\left(\left[\mathcal{V}^{*} X_{A}^{+} \mathcal{V}^{*} P_{1}\right]\right)=2 q$. Thus, if $\mathcal{V}^{*} X_{A}^{+}$is identified with $A_{1}$ and $\mathcal{V}^{*} P_{1}$ with $B_{1}$ in Lemma 2.2, it follows from (21) that $C_{A} C_{A}^{*}$ has $n-2 q$ zero eigenvalues, the remaining ones being of the form $1+\gamma_{i}(i=1: 2 q)$ with $\gamma_{i}$ the nonzero eigenvalues of $-\mathcal{V}^{*} P_{1} P_{1}^{*} \mathcal{V}+\mathcal{V}^{*} X_{A}^{+} X_{A}^{+*} \mathcal{V}$. As $q$ of these $\gamma_{i}$ are positive and the other $q$ are negative, the inequalities in (17) follow, as desired.

To prove the statement of the theorem for $q=1$, we observe that in this case $L$ is a column vector and that the Krylov matrix can be rewritten as $\mathcal{K}_{m}=$ $L^{(1)} W_{m}$, where $L^{(1)}=\operatorname{diag}\left(L_{1,1}, \ldots, L_{n, 1}\right)$ is nonsingular since, by assumption $\left|L_{j, 1}\right|=1, j=1: n$. From this observation and pseudo-inverse properties, it is immediate to see that $P_{1}$ reduces to $p_{1}, X_{A}^{+}$reduces to $x^{+}$, and neither depend on $L$. Hence it follows that $C_{A}(m, 1) C_{A}(m, 1)^{*}$ does not depend on $L$ and that

$$
C_{A}(m, 1) C_{A}^{*}(m, 1)=I_{n}-\breve{\mathcal{V}}^{*} p_{1} p_{1}^{*} \breve{\mathcal{V}}+\breve{\mathcal{V}}^{*} x^{+} x^{+*} \breve{\mathcal{V}}
$$

The equalities (18) follow on analyzing the eigenvalues of $C_{A}(m, 1) C_{A}^{*}(m, 1)$ from this equality; details can be found in [5].

Remark 1. The rank condition on $\left[Z^{m} L L\right]$ is no serious restriction in practice. This is because in practical problems $L$ is dense, in which case one can prove, under mild conditions, that $\operatorname{rank}\left(\left[Z^{m} L L\right]\right)=2 q$.

Remark 2. Theorem 2.3 generalizes one concerning the singular values of a particular projected companion matrix by Bazan (see, Thm. 4 in [5]), and shows also that the singular values of the projected block companion matrix in our context, inherits to some extent the singular value properties of general block companion matrices described in Lemma 2.7 in [15].

Since the singular values of $C_{A}(m, 1)$ do not depend on the matrix $L$, we can always compare the singular values of $C_{A}(m, q)$ for the case where $q>1$ with those corresponding to $q=1$. This is given in the following theorem. 
Theorem 2.4. Let $C_{A}(m, q)$ as before. Then, for $m \geq n$ and $1<q \leq 2 n$, there holds

$$
\sigma_{1}\left(C_{A}(m, q)\right) \leq \sigma_{1}\left(C_{A}(m, 1)\right), \quad \sigma_{n}\left(C_{A}(m, 1)\right) \leq \sigma_{n}\left(C_{A}(m, q)\right) .
$$

Proof. We shall prove the inequalities (23) for $q=2$; the proof for the case $q>2$ is similar. Notice that for $q=1$, we have

$$
C_{A}(m, 1) C_{A}^{*}(m, 1)=I_{n}+\breve{V}^{*} x^{+} x^{+*} \breve{V}-\breve{V}^{*} p_{1} p_{1}^{*} \breve{V} \doteq I_{n}+\mathcal{E}
$$

while if $q=2$, we have from (21)

$$
\begin{aligned}
C_{A}(m, q) C_{A}^{*}(m, q)= & I_{n}+\mathcal{V}^{*} X_{1} X_{1}^{*} \mathcal{V}-\mathcal{V}^{*} P_{1}^{1} P_{1}^{1^{*}} \mathcal{V} \\
& +\mathcal{V}^{*} X_{2} X_{2}^{*} \mathcal{V}-\mathcal{V}^{*} P_{1}^{2} P_{1}^{2^{*}} \mathcal{V}
\end{aligned}
$$

where we have assumed that $X_{A}^{+}=\left[X_{1}, X_{2}\right], P_{1}=\left[P_{1}^{1}, P_{1}^{2}\right]$. The idea behind the proof is to rewrite (25) in terms of the matrix $\mathcal{E}$ introduced in (24). For this we use the fact that

$$
\mathcal{V}^{*} X_{i}=\mathcal{T}_{i} \breve{V}^{*} x^{+}, \quad \mathcal{V}^{*} P_{1}^{i}=\mathcal{T}_{i} \breve{V}^{*} p_{1}, \quad i=1,2
$$

where $\mathcal{T}_{i}=\mathcal{V}^{*} \mathcal{I}_{i} \breve{\mathcal{V}}, \mathcal{I}_{1}=\left[\begin{array}{llll}e_{1} & e_{3} & \cdots & e_{2 m-1}\end{array}\right], \mathcal{I}_{2}=\left[\begin{array}{llll}e_{2} & e_{4} & \cdots & e_{2 m}\end{array}\right]$, in which $e_{i}$ denotes the $i$ th canonical vector in $\mathbb{R}^{m q}$. This can be seen as follows. Let $L=\left[L_{1}, L_{2}\right]$ and $R_{1}=\operatorname{diag}\left(L_{1,1}, \cdots, L_{n, 1}\right)$. Since $Z$ and $R_{1}$ are diagonal, the definition of $X_{1}$ implies (see (22))

$$
X_{1}=\mathcal{K}_{m}^{\dagger} Z^{m} L_{1}=\mathcal{K}_{m}^{\dagger} Z^{m} R_{1} e=\mathcal{K}_{m}^{\dagger} R_{1} W_{m} W_{m}^{\dagger} Z^{m} e=\mathcal{K}_{m}^{\dagger} R_{1} W_{m} x^{+} .
$$

But since $R_{1} W_{m}=\left[R_{1} e R_{1} Z e \cdots R_{1} Z^{m-1} e\right]$ and $\mathcal{P}=\mathcal{K}_{m}^{\dagger} \mathcal{K}_{m}$, we have

$$
\mathcal{K}_{m}^{\dagger} R_{1} W=\left[\begin{array}{lllll}
\mathcal{K}_{m}^{\dagger} L_{1} & \mathcal{K}_{m}^{\dagger} \Lambda L_{1} \cdots & \mathcal{K}_{m}^{\dagger} \Lambda^{m-1} L_{1}
\end{array}\right]=\left[\begin{array}{lllll}
\mathcal{P} e_{1} & \mathcal{P} e_{3} & \cdots & \mathcal{P} e_{2 m-1}
\end{array}\right] .
$$

Inserting this result in Eq. (27) yields

$$
X_{1}=\left[\begin{array}{llll}
\mathcal{P} e_{1} & \mathcal{P} e_{3} & \cdots & \mathcal{P} e_{2 m-1}
\end{array}\right] x^{+} .
$$

A similar work with $X_{2}, P_{1}^{1}$, and $P_{1}^{2}$ gives

$$
\begin{aligned}
X_{2} & =\left[\begin{array}{llll}
\mathcal{P} e_{2} & \mathcal{P} e_{4} & \cdots & \mathcal{P} e_{2 m}
\end{array}\right] x^{+}, \\
P_{1}^{1} & =\left[\begin{array}{llll}
\mathcal{P} e_{1} & \mathcal{P} e_{3} & \cdots & \mathcal{P} e_{2 m-1}
\end{array}\right] p_{1}, \\
P_{1}^{2} & =\left[\begin{array}{llll}
\mathcal{P} e_{2} & \mathcal{P} e_{4} & \cdots & \mathcal{P} e_{2 m}
\end{array}\right] p_{1} .
\end{aligned}
$$


The set of equations (26) follows on multiplying by $\mathcal{V}^{*}$ on both sides of equations (28), (29), (30), and (31). Here we have used the fact that $\breve{\mathcal{V}} \breve{V}^{*} x^{+}=x^{+}$, $\breve{\mathcal{V}} \breve{V}^{*} p_{1}=p_{1}$, since both $x^{+}$and $p_{1}$ belong to $\mathcal{R}\left(W_{m}^{*}\right)$.

We turn now to the proof of the theorem. Using the Eq. (26) and (24), we have

$$
C_{A}(m, q) C_{A}^{*}(m, q)=I_{n}+\mathcal{T}_{1} \mathcal{E} \mathcal{T}_{1}^{*}+\mathcal{T}_{2} \mathcal{E} \mathcal{T}_{2}^{*}
$$

Let $u$ be a unit vector in $\mathbb{C}^{p}$ and define $w_{i}$ to be the unit vector with the same direction as $\mathcal{T}_{i}^{*} u, i=1,2$. Forming the Rayleigh-Ritz quotient in (32), we have

$$
\begin{aligned}
u^{*} C_{A}(m, q) C_{A}^{*}(m, q) u & =1+w_{1}^{*} \mathcal{E} w_{1}\left\|\mathcal{T}_{1}^{*} u\right\|^{2}+w_{2}^{*} \mathcal{E} w_{2}\left\|\mathcal{T}_{2}^{*} u\right\|^{2} \\
& \leq 1+w^{*} \mathcal{E} w\left(\left\|\mathcal{T}_{1}^{*} u\right\|^{2}+\left\|\mathcal{T}_{2}^{*} u\right\|^{2}\right),
\end{aligned}
$$

where $w=w_{i}$ such that $w^{*} \mathcal{E} w=\max \left\{w_{1}^{*} \mathcal{E} w_{1}, w_{2}^{*} \mathcal{E} w_{2}\right\}$. Now using the definition of matrix $\mathcal{T}_{1}$, we have

$$
\left\|\mathcal{T}_{1}^{*} u\right\|^{2}=u^{*} \mathcal{T}_{1} \mathcal{T}_{1}^{*} u=u^{*} \mathcal{V}^{*} \mathcal{I}_{1} \breve{\mathcal{V}} \breve{\mathcal{V}}^{*} \mathcal{I}_{1}^{*} \mathcal{V} u=\left\|\breve{\mathcal{P}} \mathcal{I}_{1}^{*} \mathcal{V} u\right\|^{2} \leq\left\|\mathcal{I}_{1}^{*} \mathcal{V} u\right\|^{2}
$$

where we have used the fact that $\breve{\mathcal{P}} \breve{\mathcal{P}}^{*}=\breve{\mathcal{V}} \breve{V}^{*}$. A similar work gives

$$
\left\|\mathcal{T}_{2}^{*} u\right\|^{2} \leq\left\|\mathcal{I}_{2}^{*} \mathcal{V} u\right\|^{2}
$$

Summing up the two last inequalities it is not difficult to check that

$$
\left\|\mathcal{T}_{1}^{*} u\right\|^{2}+\left\|\mathcal{T}_{2}^{*} u\right\|^{2} \leq 1
$$

Substituting this result in (33) gives

$$
u^{*} C_{A}(m, q) C_{A}^{*}(m, q) u \leq w^{*}(I+\mathcal{E}) w \leq \sigma_{\max }^{2}\left(C_{A}(m, 1)\right),
$$

and the proof of the first inequality in (23) is concluded.

Finally, since $\sigma_{n}\left(C_{A}(m, q)\right)=1 / \sigma_{1}\left(C_{B}(m, q)\right)$, by Lemma (2.1), proceeding as before it follows $\sigma_{1}\left(C_{B}(m, q)\right) \leq \sigma_{1}\left(C_{B}(m, 1)\right)$. This proves the second inequality in (23) and the proof of the theorem is concluded.

A point that remains for discussion is the behavior of the singular values of $C_{A}(m, q)$ for fixed $q \geq 1$ and varying $m$. This is a difficult problem; so we restrict ourselves to analyzing bounds for them. 
Corollary 2.5 Let $X_{A}^{+}$and $P_{1}$ be as in (22). Then we have

$$
\sqrt{1-\left\|P_{1}\right\|_{2}^{2}} \leq \sigma_{n}\left(C_{A}\right), \quad \sigma_{1}\left(C_{A}\right) \leq \sqrt{1+\left\|X_{A}^{+}\right\|_{2}^{2}} .
$$

Additionally, while the lower bound increases with $m$, the upper bound decreases.

Proof. First notice from (21) that the squared singular values of $C_{A}$ that differ from 1 are the eigenvalues of $\Omega$ defined by

$$
\begin{aligned}
\Omega & =I_{2 q}+\left[\begin{array}{c}
X_{A}^{+*} \mathcal{V} \\
-P_{1}^{*} \mathcal{V}
\end{array}\right]\left[\mathcal{V}^{*} X_{A}^{+}\right. \\
& \left.\mathcal{V}^{*} P_{1}\right] \\
& =\left[\begin{array}{cc}
I_{q}+X_{A}^{+*} X_{A}^{+} & X_{A}^{+*} P_{1} \\
-P_{1}^{*} X_{A}^{+} & I_{q}-P_{1}^{*} P_{1}
\end{array}\right] .
\end{aligned}
$$

By comparing the eigenvalues of $\Omega$ with those of its Hermitian part, it follows

$$
\lambda_{\min }\left(I_{q}-P_{1}^{*} P_{1}\right) \leq \lambda_{i}(\Omega) \leq \lambda_{\max }\left(I_{q}+X_{A}^{+*} X_{A}^{+}\right) .
$$

This proves (34). We shall now prove that both $\left\|X_{A}^{+}\right\|_{2}$ and $\left\|P_{1}\right\|_{2}$ are decreasing functions of $m$. Let

$$
\breve{\mathcal{K}}_{m}=\left[\begin{array}{lll}
L & Z L & \cdots
\end{array} Z^{m} L\right], \quad \breve{X}_{A}^{+}=\breve{\mathcal{K}}_{m}^{\dagger} Z^{m+1} L, \quad\left\|\breve{P}_{1}\right\|_{2}=\breve{\mathcal{K}}_{m}^{\dagger} L .
$$

Then we shall prove that $\left\|\breve{X}_{A}^{+}\right\|_{2} \leq\left\|X_{A}^{+}\right\|_{2}$ and $\left\|\breve{P}_{1}\right\|_{2} \leq\left\|P_{1}\right\|_{2}$. In fact, write $\breve{\mathcal{K}}_{m}=\left[L \mid Z \mathcal{K}_{m}\right]$ and notice that

$$
\breve{\mathcal{K}}_{m}^{\dagger *} \breve{\mathcal{K}}_{m}^{\dagger}=\left(\breve{\mathcal{K}}_{m} \breve{\mathcal{K}}_{m}^{*}\right)^{-1}=\left(L L^{*}+Z G_{m, q} Z^{*}\right)^{-1},
$$

where

$$
G_{m, q}=\mathcal{K}_{m} \mathcal{K}_{m}^{*} .
$$

Applying the Sherman-Morrison formula to the inverse above we obtain

$$
\begin{aligned}
\breve{\mathcal{K}}_{m}^{\dagger *} \breve{\mathcal{K}}_{m}^{\dagger} & =Z^{-*} G_{m, q}^{-1} Z^{-1}-Z^{-*} G_{m, q}^{-1} Z^{-1} L\left(I+L^{*} Z^{-*} G_{m, q}^{-1} Z^{-1} L\right)^{-1} L^{*} Z^{-*} G_{m, q}^{-1} Z^{-1} \\
& =Z^{-*} \mathcal{K}_{m}^{\dagger *} \mathcal{K}_{m}^{\dagger} Z^{-1}-Z^{-*} \mathcal{K}_{m}^{\dagger *} X_{B}^{+}\left(I+X_{B}^{+*} X_{B}^{+}\right)^{-1} X_{B}^{+*} \mathcal{K}_{m}^{\dagger} Z^{-1},
\end{aligned}
$$

where we have used the fact that $\mathcal{K}_{m} \mathcal{K}_{m}^{\dagger}=I_{n}$, and we set $\breve{X}_{B}^{+}=\breve{\mathcal{K}}_{m}^{\dagger} Z^{-1} L$. Pre-multiplication by $L^{*} Z^{m+1^{*}}$ and post-multiplication by $Z^{m+1} L$ on both sides of this equation yields

$$
\breve{X}_{A}^{+*} \breve{X}_{A}^{+}=X_{A}^{+*} X_{A}^{+}-X_{A}^{+*} X_{B}^{+}\left(I+X_{B}^{+*} X_{B}^{+}\right)^{-1} X_{B}^{+*} X_{A}^{+} .
$$


This shows that the singular values of of $\breve{X}_{A}^{+}$can not exceed those of $X_{A}^{+}$, thus ensuring the statement of the theorem for $X_{A}^{+}$. To prove that $\left\|P_{1}\right\|_{2}$ decreases with $m$, it is sufficient to partition $\breve{\mathcal{K}}_{m}$ as $\breve{\mathcal{K}}_{m}=\left[\mathcal{K}_{m} \mid Z^{m} L\right]$, and then proceed as before.

The corollary is interesting because it provides a bound for the 2-norm condition number of $C_{A}$ of the form

$$
\kappa\left(C_{A}\right) \leq \frac{\sqrt{1+\left\|X_{A}^{+}\right\|_{2}^{2}}}{\sqrt{1-\left\|P_{1}\right\|_{2}^{2}}}
$$

that decreases with $m$. Thus, reliable bounds for $\kappa\left(C_{A}\right)$ can be obtained provided both $\left\|X_{A}^{+}\right\|_{2}^{2}$ and $\left\|P_{1}\right\|_{2}^{2}$ are small enough. For the significant case where the prescribed eigenvalues lie inside the unit circle, the asymptotic of the bounds as $m$ is going to infinite is readily determined. To do this the following technical result, the proof of which is straightforward, is needed.

Lemma 2.6. Suppose all $z_{j}$ fall inside the unit circle. Then $\left\|X_{A}^{+}\right\|_{2} \rightarrow 0$ as $m \rightarrow \infty$.

Corollary 2.7. Suppose all $z_{j}$ lie inside the unit circle. Then, as $m \rightarrow \infty$ we have

$$
\kappa\left(C_{A}\right) \leq \prod_{j=1}^{n}\left|z_{j}\right|^{-1} .
$$

Proof. We first notice that for $q=1$ we have $\sigma_{1}\left(C_{A}(m, 1)\right) \sigma_{n}\left(C_{A}(m, 1)\right)=$ $\prod_{j=1}^{n}\left|z_{j}\right|$. Using Corollary 2.5 and Lemma 2.6 it follows that

$$
\lim _{m \rightarrow \infty} \sigma_{1}\left(C_{A}(m, q)\right)=\lim _{m \rightarrow \infty} \sigma_{1}\left(C_{A}(m, 1)\right)=1 .
$$

Now since $\sigma_{n}\left(C_{A}(m, q)\right) \geq \sigma_{n}\left(C_{A}(m, 1)\right)$ for all $m \geq n$ and fixed $q>1$, by Corollary 2.5 again, there holds

$$
\lim _{m \rightarrow \infty} \sigma_{n}\left(C_{A}(m, q)\right) \geq \lim _{m \rightarrow \infty} \sigma_{n}\left(C_{A}(m, 1)\right)=\prod_{j=1}^{p}\left|z_{j}\right| .
$$

The assertion of the corollary follows on using this inequality and the definition of $\kappa\left(C_{A}\right)$. 


\section{Departure from normality of $C_{A}(m, q)$}

The influence of nonnormality on several problems in scientific computing has been known for long time and several measures of nonnormality either of theoretical or practical interest are now available [8, 10, 13]. An exhaustive discussion on the influence of nonnormality on many problems in scientific computing, using several measures of nonnormality, is given in Chaitin-Chatelin and Frayseé [8]. For $A \in \mathbb{C}^{n \times n}$ the following measure has been introduced by Henrici (1962):

$$
D^{2}(A)=\|A\|_{F}^{2}-\sum_{j=1}^{n}\left|\lambda_{j}(A)\right|^{2} .
$$

This measure plays an important role in our context because it can be related to the conditioning of the eigenbasis of $A$ when $A$ is diagonalizable. To clarify this recall that for general $A \in \mathbb{C}^{n \times n}$ with simple eigenvalues $\lambda_{j}$ and $u_{j}, v_{j}$ as associated left and right eigenvectors, the condition number of $\lambda_{j}$, denoted by $\kappa_{j}\left(\lambda_{j}\right)$, is defined by (see. e.g., Wilkinson [30, p. 314])

$$
\kappa\left(\lambda_{j}\right)=\frac{\left\|u_{j}\right\|_{2}\left\|v_{j}\right\|_{2}}{\left|u_{j}^{*} v_{j}\right|} .
$$

Smith [23] proved that

$$
\kappa_{j}\left(\lambda_{j}\right) \leq\left[1+\frac{1}{n-1}\left(\frac{D}{\delta_{j}}\right)^{2}\right]^{(n-1) / 2},
$$

where $\delta_{j}$ measures the distance of $\lambda_{j}$ to the rest of the spectrum. Thus the more the ill-conditioned $\lambda_{j}$, the larger the ratio $D / \delta_{j}$, which means that $D$ increases and/or $\delta_{j}$ is small. Another interpretation of the above result is possible. Of course, it says that for the eigenvalue $\lambda_{j}$ to be well conditioned, it suffices that $D / \delta_{j} \approx 0$ and $n$ be a moderate number. We shall return to this point later.

The goal here is to analyze $D\left(C_{A}(m, q)\right)$, concentrating on its behavior as a function of $m, q$ for fixed $q \geq 1$ and increasing $m$. The following theorem shows that this can be made by comparing the singular values of $C_{A}(m, q)$ with those of $C_{A}(m, 1)$. This is always possible, since by Theorem 2.3, the singular values of $C_{A}(m, 1)$ do not depend on the matrix $L$. 
Theorem 3.1. Let $a$ and $b$ denote respectively the largest and the smallest singular values of $C_{A}(m, 1)$ and let the singular values $\sigma_{j}$ of $C_{A}(m, q)$ be ordered in the usual way, i.e., $\sigma_{1} \geq \sigma_{2} \geq \cdots \geq \sigma_{n}$. Let

$$
\begin{aligned}
\sigma^{*} & =\max \left\{\frac{\sigma_{1}}{a}, \sigma_{2}, \cdots, \sigma_{n-1}, \frac{\sigma_{n}}{b}\right\}, \text { and } \\
\sigma_{*} & =\min \left\{\frac{\sigma_{1}}{a}, \sigma_{2}, \cdots, \sigma_{n-1}, \frac{\sigma_{n}}{b}\right\} .
\end{aligned}
$$

Define

$$
\hat{k}=\frac{\sigma^{*}}{\sigma_{*}}, \quad \rho=\left[\frac{a^{2}-\sigma_{1}^{2}}{a^{2}}\left(a^{2}-1\right)+\frac{\sigma_{n}^{2}-b^{2}}{b^{2}}\left(1-b^{2}\right)\right] .
$$

Then, for each $m \geq n$ and $1<q \leq n / 2$ it holds

$$
\begin{aligned}
\left\|C_{A}(m, 1)\right\|_{F}^{2}-\rho & \leq\left\|C_{A}(m, q)\right\|_{F}^{2} \\
& \leq \frac{q}{2}\left(\hat{k}+\hat{k}^{-1}\right)^{2}-(\rho+2 q)+\left\|C_{A}(m, 1)\right\|_{F}^{2} .
\end{aligned}
$$

Proof. We first notice that, because of Theorem (2.3), we have

$$
\left\|C_{A}(m, 1)\right\|_{F}^{2}=n-2+a^{2}+b^{2} .
$$

Now since $C_{A}(m, q)$ has the same spectrum as $C_{A}(m, 1)$ we have

$$
\sigma_{1}^{2} \sigma_{2}^{2} \cdots \sigma_{q}^{2} \sigma_{n-q+1}^{2} \cdots \sigma_{n-1}^{2} \sigma_{n}^{2}=\prod_{j=1}^{n}\left|z_{j}\right|^{2}=a^{2} b^{2} .
$$

If this is rewritten as

$$
1=\frac{a^{2}}{\sigma_{1}^{2}} \frac{1}{\sigma_{2}^{2}} \cdots \frac{1}{\sigma_{q}^{2}} \frac{1}{\sigma_{n-q+1}^{2}} \frac{1}{\sigma_{n-q+2}^{2}} \cdots \frac{1}{\sigma_{n-1}^{2}} \frac{b^{2}}{\sigma_{n}^{2}},
$$

the geometric-arithmetic mean inequality leads to

$$
2 q \leq \frac{a^{2}}{\sigma_{1}^{2}}+\frac{1}{\sigma_{2}^{2}}+\cdots+\frac{1}{\sigma_{p}^{2}}+\frac{1}{\sigma_{n-p+1}^{2}}+\cdots+\frac{1}{\sigma_{n-1}^{2}}+\frac{b^{2}}{\sigma_{n}^{2}} .
$$

Multiplying both sides of this inequality by the sum of the reciprocals of each term of the right hand side, we obtain

$$
\left[\frac{\sigma_{1}^{2}}{a^{2}}+\sigma_{2}^{2}+\cdots+\sigma_{q}^{2}+\sigma_{n-p+1}^{2}+\cdots+\sigma_{n-1}^{2}+\frac{\sigma_{n}^{2}}{b^{2}}\right] 2 q \leq c^{*} A c c^{*} A^{-1} c,
$$


where

$$
\begin{aligned}
A & =\operatorname{diag}\left(\sigma_{1}^{2} / a^{2}, \sigma_{2}^{2}, \ldots, \sigma_{q}^{2}, \sigma_{n-q+1}^{2}, \ldots, \sigma_{n-1}^{2}, \sigma_{n}^{2} / b^{2}\right), \\
c & =[1,1, \ldots, 1]^{T} \in \mathbb{R}^{2 q} .
\end{aligned}
$$

Kantorovic's inequality (see Horn and Johnson [16, Thm. 7.4.41]) leads then to

$$
\left[\frac{\sigma_{1}^{2}}{a^{2}}+\sigma_{2}^{2}+\cdots+\sigma_{q}^{2}+\sigma_{n-p+1}^{2}+\cdots+\sigma_{n-1}^{2}+\frac{\sigma_{n}^{2}}{b^{2}}\right] 2 q \leq\left[q\left(\hat{k}+\hat{k}^{-1}\right)\right]^{2},
$$

where $\hat{k}$ is defined in (42). Hence it follows

$$
\sigma_{1}^{2}+\cdots+\sigma_{n}^{2} \leq \frac{q}{2}\left(\hat{k}+\hat{k}^{-1}\right)^{2}+\sigma_{1}^{2}-\frac{\sigma_{1}^{2}}{a^{2}}+\sigma_{n}^{2}-\frac{\sigma_{n}^{2}}{b^{2}}+n-2 q .
$$

The upper bound in (43) follows from this inequality on noting that

$$
\sigma_{1}^{2}-\frac{\sigma_{1}^{2}}{a^{2}}+\sigma_{n}^{2}-\frac{\sigma_{n}^{2}}{b^{2}}=-\rho+a^{2}+b^{2}-2,
$$

where $\rho$ is defined in (42). To prove the lower bound, rewrite (45) as

$$
1=\frac{\sigma_{1}^{2}}{a_{1}} \sigma_{2}^{2} \cdots \sigma_{q}^{2} \sigma_{n-q+1}^{2} \sigma_{n-q+2}^{2} \cdots \sigma_{n-1}^{2} \frac{\sigma_{n}^{2}}{b^{2}} .
$$

The geometric-arithmetic mean inequality leads then to

$$
2 q \leq \frac{\sigma_{1}^{2}}{a_{1}^{2}}+\sigma_{2}^{2}+\cdots+\sigma_{q}^{2}+\sigma_{n-q+1}^{2}+\sigma_{n-q+2}^{2}+\cdots+\sigma_{n-1}^{2}+\frac{\sigma_{n}^{2}}{b^{2}}
$$

The lower bound in (43) is a consequence of using (47) in this inequality.

The departure from normality of $C_{A}(m, 1)$ is analyzed in Bazan [5]. The conclusion drawn from that analysis is that this matrix becomes close to a normal matrix provided the eigenvalues $z_{j}$ fall near the unit circle and $m$ is large enough. This is important in our context since if we take into account the inequalities (43), we can conclude that $C_{A}(m, q)$ for the case $q>1$ may become closer to normality than $C_{A}(m, 1)$. In terms of eigenvalue sensitivity, this means that prescribed eigenvalues of $P_{m}(z)$ can be less sensitive to noise when regarded as eigenvalues of $C_{A}(m, q)$ with $q>1$ than when regarded as eigenvalues of $C_{A}(m, 1)$. This shall be theoretically demonstrated in the next section. Here we restrict ourselves to numerically illustrate the behavior of $D\left(C_{A}(m, q)\right)$. 
Example: departure from normality of $C_{A}(m, q)$ arising from a dynamical system. The dynamical system under analysis is defined by the state space equations

$$
\begin{aligned}
& \dot{x}=A x+B u \\
& y=C x,
\end{aligned}
$$

and corresponds to a computer model of a flexible structure known as MiniMast [18]. Matrices $A, B$ and $C$ are of orders $10 \times 10,10 \times 2$ and $2 \times 10$, respectively; the entries of the matrices can be found in [18]. Impulse response samples are thus given as

$$
h_{k}=C e^{A \Delta t k} B, k=0,1, \ldots
$$

Matrices $R$ and $L$ of model (3) are thus of order $2 \times 10$ and $10 \times 2$, respectively, and can be found readily by computing an eigendecomposition of matrix $A$. According to our notation this implies that $n=10, p=q=2$; the eigenvalues are of the form $z_{j}=e^{s_{j} \Delta t}(j=1: 10)$ where the $s_{j}$ 's are eigenvalues of $A$. The time step is $\Delta t=0.03 \mathrm{~s}$. The model comprises five modes (in complex conjugate pairs) and involves two closely spaced frequency pairs. Frequencies and damping expressed as the negative real part of the $z_{j}$ 's as well as the eigenvalues in modulus and separations $\delta_{j}=\min \left|z_{j}-z_{i}\right|, i \neq j$, are displayed in Table 1 .

\begin{tabular}{c|c|c|c|c}
\hline $\begin{array}{c}\text { Mode } \\
j\end{array}$ & Damping & $\begin{array}{c}\text { Frequency } \\
\mathrm{rad} / \mathrm{s}\end{array}$ & $\left|z_{j}\right|$ & $\delta_{j}$ \\
\hline 1 & 0.32907 & 27.42011 & 0.99017 & 0.32299 \\
2 & 0.38683 & 38.68230 & 0.98846 & 0.00982 \\
3 & 0.38352 & 38.35103 & 0.98856 & 0.00982 \\
4 & 0.09066 & 5.03555 & 0.99728 & 0.00011 \\
5 & 0.09055 & 5.03176 & 0.99728 & 0.00011 \\
\hline
\end{tabular}

Table 1 - System poles $z_{j}$ and separations.

In order to illustrate the behavior of $D^{2}\left(C_{A}(m, q)\right)$ as a function of $m, q$ the norms $\left\|C_{A}(m, q)\right\|_{F}^{2}$ for increasing $m$ and $q=1: 2$ were computed from the relation (see (21))

$$
\left\|C_{A}(m, q)\right\|_{F}^{2}=n+\left\|X_{A}^{+}\right\|_{F}^{2}-\left\|P_{1}\right\|_{F}^{2} .
$$




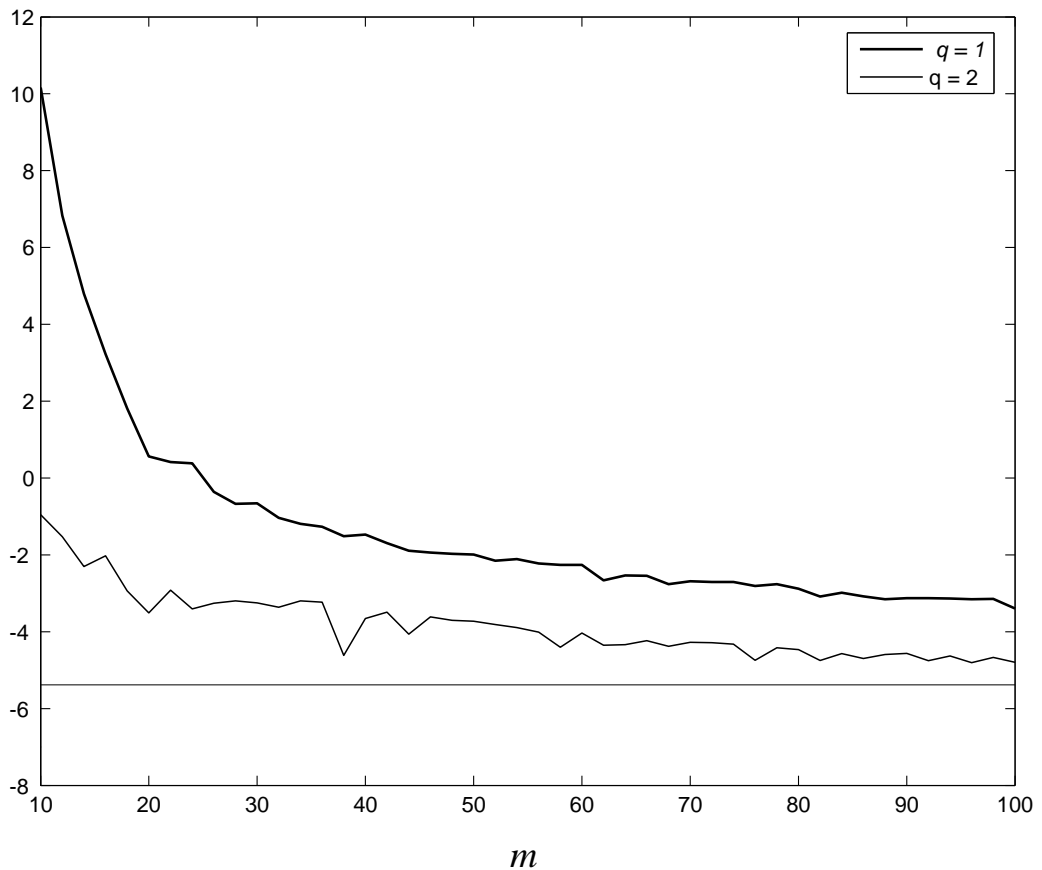

Figure 1 - Departure from normality of matrix $C_{A}(m, q)$ as a function of $m$ and $q$ on a logarithmic scale; $q$ denotes the number of columns of matrix $L$ in (3) and $m$ the degree of $P_{m}(z)$. The horizontal line points out the asymptotic value of $D^{2}\left(C_{A}(m, 2)\right)$ for large $m$.

All computations were carried out using MATLAB. The results displayed in Figure 1 are surprising: they not only show that $D^{2}\left(C_{A}(m, 2)\right)$ really improves $D^{2}\left(C_{A}(m, 1)\right)$ but also that this improvement can be dramatic when $m$ is near $n=10$. For illustration, while for $q=1$ and $m=10,11$ we obtain

$$
D^{2}\left(C_{A}(10,1)\right)=2.5877 \times 10^{4}, \quad D^{2}\left(C_{A}(11,1)\right)=3.692 \times 10^{3},
$$

which illustrate that $C_{A}(10,1)$ and $C_{A}(11,1)$ are highly nonnormal, for $q=2$ and the same values of $m$ we obtain

$$
D^{2}\left(C_{A}(10,2)\right)=5.4295 \times 10^{-1}, \quad D^{2}\left(C_{A}(11,2)\right)=4.9053 \times 10^{-1}
$$

The influence of $q$ on $D^{2}\left(C_{A}(m, q)\right)$ for $q>2$ was also analyzed. For this, input matrices $B$ with random numbers as entries of orders $q \times 10$ and $q=1: 4$ 


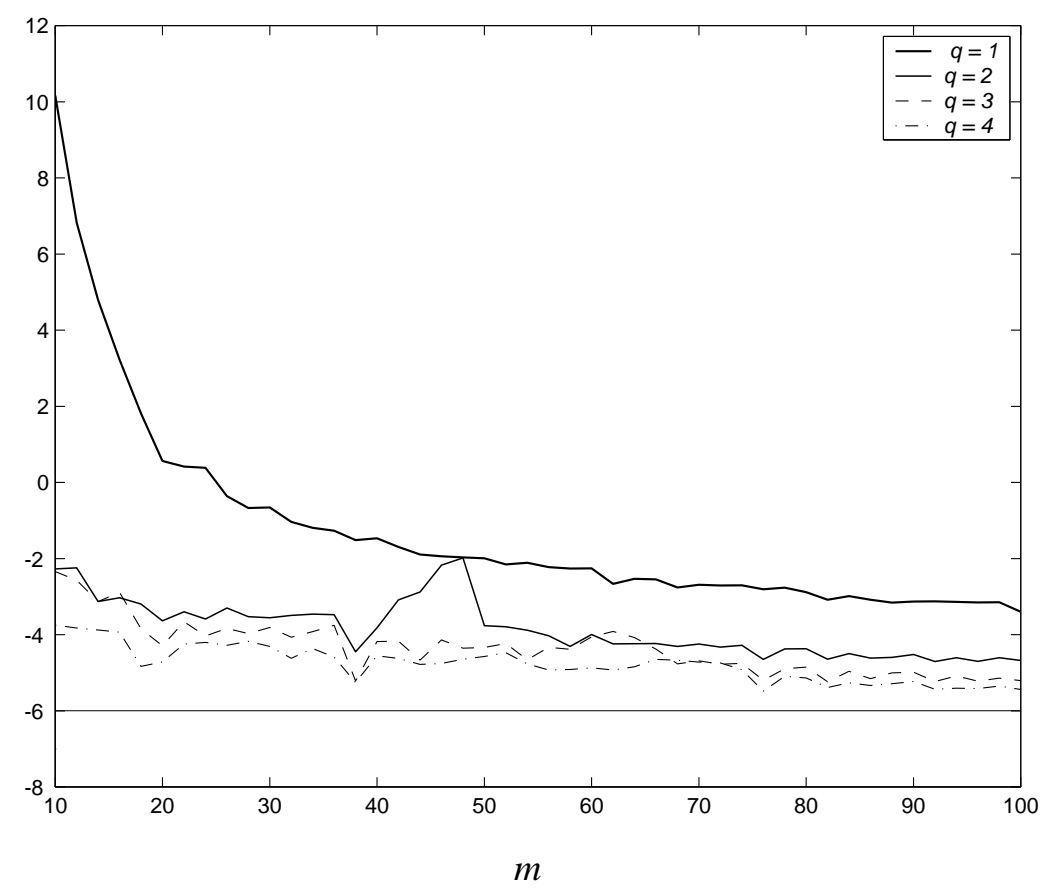

Figure 2 - Departure from normality of matrix $C_{A}(m, q)$ as a function of $m$ and $q$ on a logarithmic scale; $q$ denotes the number of columns of matrix $L$ in (3) and $m$ the degree of $P_{m}(z)$. The horizontal line points out the asymptotic value of $D^{2}\left(C_{A}(m, 4)\right)$ for large $m$.

were constructed. With these matrices at hand, the matrices $L$ of corresponding orders were obtained in the same way as in the case for $q=2$. Results corresponding to the seed value of the random generator equal to 10 (we use the MATLAB function randn), displayed in Figure 2, show once more that the departure from normality of matrix $C_{A}(m, q)$ for $q>1$ gets smaller than that corresponding to $q=1$. However no conclusion can be drawn concerning the behavior of $D^{2}\left(C_{A}(m, q)\right)$ for values $q>2$ in comparison with that corresponding to $q=2$.

As in this example all eigenvalues lie inside the unit circle, the asymptotic value of $D^{2}\left(C_{A}(m, q)\right)$ as $m$ is going to infinity can be readily computed: it suffices to use (49) taking into account that in this case

$$
\left\|X_{A}\right\|_{F}^{2} \rightarrow 0, \quad\left\|P_{1}\right\|_{F}^{2}=\operatorname{Trace}\left(L^{*} G_{\infty, q}^{-1} L\right)
$$


where

$$
\mathcal{G}_{\infty, q}=\lim _{m \rightarrow \infty} \mathcal{G}_{m, q}=\lim _{m \rightarrow \infty} \mathcal{K}_{m} \mathcal{K}_{m}^{*} \text {, with }\left[\mathcal{G}_{m, q}\right]_{i, j}=e_{i}^{*} L L^{*} e_{j} \frac{1-\left(z_{i} \bar{z}_{j}\right)^{m}}{1-z_{i} \bar{z}_{j}} .
$$

Asymptotic values of $D^{2}\left(C_{A}(m, q)\right)$ in this case are:

$$
\begin{array}{ll}
D^{2}\left(C_{A}(\infty, 1)\right)=0.9719 \times 10^{-2}, & D^{2}\left(C_{A}(\infty, 2)\right)=0.4915 \times 10^{-2}, \\
D^{2}\left(C_{A}(\infty, 3)\right)=0.2946 \times 10^{-2}, & D^{2}\left(C_{A}(\infty, 4)\right)=0.2489 \times 10^{-2}
\end{array}
$$

\section{Condition numbers}

We have seen that the prescribed eigenvalues $z_{j}$ of $P_{m}(z)$ are eigenvalues of the projected companion matrix $C_{A}(m, q)$. This fact is exploited here to carry out a sensitivity analysis of these eigenvalues. To this end, we choose as measures of sensitivity the Wilkinson condition numbers of the $z_{j}$ 's (see (40)) viewed as eigenvalues of $C_{A}(m, q)$, and the overall 2-norm condition number of the eigenvalue problem. In order to describe our results we recall that for $m \geq n$ and fixed $q, q \geq 1, \mathcal{G}_{m, q}=\mathcal{K}_{m} \mathcal{K}_{m}^{*}$ is positive definite Hermitian. In the sequel we shall always assume that the left eigenvector of $P_{m}(z)$ (the rows of matrix $L$ in (3)) are scaled using the 2-norm to unit length. The lemma below explains that the sensitivity of the eigenvalue problem associated with matrix $C_{A}(m, q)$ is governed by the condition number of matrix $G_{m, q}$.

Lemma 4.1. Let $C_{A}(m, q)$ be as before. Then there holds

$$
C_{A}(m, q)=\left(G_{m, q}\right)^{-1 / 2} Z\left(G_{m, q}\right)^{1 / 2} .
$$

Consequently, the sensitivity of the eigenvalue problem related to the prescribed eigenvalues is governed by $\sqrt{\kappa\left(G_{m, q}\right)}$.

Proof. Set $\mathcal{V}=\mathcal{K}_{m}^{*}\left(G_{m, q}\right)^{-1 / 2}$. It is immediate to check that the columns of $\mathcal{V}$ form an orthonormal basis of $\mathcal{R}\left(\mathcal{K}_{m}^{*}\right)$. Using the definition of $C_{A}(m, q)$ and this basis, we have

$$
\begin{aligned}
C_{A}(m, q) & =\mathcal{V}^{*} C_{A} \mathcal{V} \\
& =\left(G_{m, q}\right)^{-1 / 2} \mathcal{K}_{m} C_{A} \mathcal{K}_{m}^{*}\left(G_{m, q}\right)^{-1 / 2}
\end{aligned}
$$

The proof concludes on using (8). 
In the following, the condition number of $z_{j}$ related to $C_{A}(m, q)$ for $q>1$ (and hence to $P_{m}(z)$ ) is denoted by $\kappa_{q}\left(z_{j}\right)$, while the condition number of the same eigenvalue but related to $C_{A}(m, 1)$ is denoted by $\kappa_{1}\left(z_{j}\right)$.

Theorem 4.2. For $m \geq n$ the following properties hold

(a) For $1 \leq q \leq n / 2$ we have

$$
\kappa_{q}\left(z_{j}\right)=\sqrt{1+\left|z_{j}\right|^{2}+\left|z_{j}\right|^{4}+\cdots+\left|z_{j}\right|^{2(m-1)}}\left\|\mathcal{K}_{m}^{\dagger} e_{j}\right\|_{2} \quad(j=1: n) .
$$

(b) The condition numbers $\kappa_{1}\left(z_{j}\right)$ do not depend on the matrix $L$ but rather on the Vandermonde matrix $W_{m}$.

(c) For fixed $m \geq n$ and $q>1$ there holds $\kappa_{q}\left(z_{j}\right) \leq \kappa_{1}\left(z_{j}\right)$.

(d) Let $\delta_{j}=\min _{\substack{1 \leq k \leq n \\ 1 \leq j \leq n, j \neq k}}\left|z_{j}-z_{k}\right|$ Then, for $1 \leq q \leq n / 2$ there holds

$$
\kappa_{q}\left(z_{j}\right) \leq\left[1+\frac{n-1+\left\|x^{+}\right\|_{2}^{2}+\prod_{j=1}^{n}\left|z_{j}\right|^{2}-\sum_{j=1}^{n}\left|z_{j}\right|^{2}}{(n-1) \delta_{j}^{2}}\right]^{(n-1) / 2},
$$

where $x^{+}$denotes the minimum norm solution of the system (5) for the case $q=1$.

Proof. To prove (a) notice from Lemma 4.1 that $v_{j}=G_{m, q}^{-1 / 2} e_{j}$ and $u_{j}=G_{m, q}^{1 / 2} e_{j}$ are left and right eigenvectors of $C_{A}(m, q)$, respectively, associated with the eigenvalue $z_{j}$. These eigenvectors satisfy the condition $u_{j}^{*} v_{j}=1$. Besides this

$$
\left\|v_{j}\right\|_{2}^{2}=v_{j}^{*} v_{j}=e_{j}^{*} G_{m, q}^{-1} e_{j}=\left\|\mathcal{K}_{m}^{\dagger} e_{j}\right\|_{2}^{2},
$$

and

$$
\left\|u_{j}\right\|_{2}^{2}=e_{j}^{*} G_{m, q} e_{j}=\left\|\mathcal{K}_{m}^{*} e_{j}\right\|_{2}^{2}=1+\left|z_{j}\right|^{2}+\left|z_{j}\right|^{4}+\cdots+\left|z_{j}\right|^{2(m-1)} .
$$

The last equality is because the rows of $L$ in (3) are scaled to unit length by assumption. The equality (50) follows from these relations on using the definitions given in (40).

To prove property $(b)$ notice that $L$ becomes a column vector in $\mathbb{C}^{n}$ when $q=1$. In this case we can write $\mathcal{G}_{m, q}=L^{(1)} W_{m} W_{m}^{*} L^{(1) *}$ where $L^{(1)}$ denotes a diagonal 
matrix with the components of $L$ as entries and $W_{m}$ the Vandermonde matrix introduced in the previous section. From this observation and the definition (40) it is immediate that

$$
\kappa_{1}\left(z_{j}\right)=\left\|e_{j}^{*} W_{m}\right\|_{2}\left\|W_{m}^{\dagger} e_{j}\right\|_{2},
$$

which proves $(b)$.

The proof of $(c)$ is based on the property that $\left\|\mathcal{K}_{m}^{\dagger} e_{j}\right\|_{2} \leq\left\|W_{m}^{\dagger} e_{j}\right\|_{2}$, which can be seen as follows. Let $\phi_{j}=\mathcal{K}_{m}^{\dagger} e_{j}$. This means that $\phi_{j}$ is the minimum 2-norm solution of the underdetermined linear system

$$
\mathcal{K}_{m} \phi=e_{j}
$$

Let $\widetilde{\mathcal{K}}_{m}=\left[L^{(1)} W_{m} \cdots L^{(q)} W_{m}\right]$, where $L^{(i)}=\operatorname{diag}\left(L_{1, i}, \ldots L_{n, i}\right), i=1 \ldots q$. It is clear that $\tilde{\mathcal{K}}_{m}=\mathcal{K}_{m} \mathcal{J}$ with $\mathcal{J}$ an appropriate permutation matrix. Introduce $\tilde{\mathcal{K}}_{m}^{D}$ defined by

$$
\tilde{\mathcal{K}}_{m}^{D}=\left[\begin{array}{c}
W_{m}^{\dagger} L^{(1)} \\
\vdots \\
W_{m}^{\dagger} L^{(q)}
\end{array}\right] .
$$

Then

$$
\tilde{\mathcal{K}}_{m} \tilde{\mathcal{K}}_{m}^{D}=L^{(1)} L^{(1) *}+\cdots L^{(q)} L^{(q) *}=I_{n},
$$

and therefore $\tilde{\mathcal{K}}_{m}^{D}$ is a right inverse of $\widetilde{\mathcal{K}}_{m}$. Define now $\phi=\mathcal{J} \tilde{\mathcal{K}}_{m}^{D} e_{j}$. It is not difficult to check that this vector is a solution of the system (52). Additionally

$$
\begin{aligned}
\|\phi\|_{2}^{2} & =\left\|W_{m} e_{j}\right\|_{2}^{2}\left|L_{j, 1}\right|^{2}+\left\|W_{m} e_{j}\right\|_{2}^{2}\left|L_{j, 2}\right|^{2}+\cdots+\left\|W_{m} e_{j}\right\|_{2}^{2}\left|L_{j, q}\right|^{2} \\
& =\left\|W_{m} e_{j}\right\|_{2}^{2}\left(\left|L_{j, 1}\right|^{2}+\left|L_{j, 2}\right|^{2}+\cdots+\left|L_{j, q}\right|^{2}\right) \\
& =\left\|W_{m} e_{j}\right\|_{2}^{2} .
\end{aligned}
$$

This equality proves property (c) as $\phi_{j}$ is the solution of minimum norm of (52).

Finally, property $(d)$ is a consequence of estimate (41), property (c), and Lemma 7 in Bazán [5] where it is proved that

$$
D^{2}\left(C_{A}(m, 1)\right) \leq n-1+\left\|x^{+}\right\|_{2}^{2}+\prod_{j=1}^{n}\left|z_{j}\right|^{2}-\sum_{j=1}^{n}\left|z_{j}\right|^{2} .
$$

The main conclusion that can be drawn from the Theorem 4.2 is that the sensitivity of the $z_{j}$ 's regarded as eigenvalues of the projected companion matrix essentially 
depends on intrinsic characteristics of the eigenvalues themselves and on the degree of the associated matrix polynomial. Concerning the estimates (51), since $n$ is assumed to be small, the conclusion is that they can approach the optimum value 1 provided $\left\|x^{+}\right\|_{2}^{2} \approx 0$ and the eigenvalues in modulus are reasonably close to the unit circle but not extremely close to each other. In spite of the fact that this conclusion seems to emerge under rather stringent conditions, namely, $n$ small and $z_{j}$ 's close to the unit circle, we emphasize that there are many applications in which these conditions appear frequently. In fact, in modal analysis of vibrating structures, the analysis of slow-decaying signals often involves eigenvalues very close to the unit circle and $n$ small; in $[4,1,19]$ examples are reported with $n$ ranging from 15 to 20 . Numerical examples showing that $\left\|x^{+}\right\|_{2}^{2} \approx 0$ for moderate values of $m$ are discussed in [7]. Another example involving the condition $n$ small is encountered in NMR; genuine applications in this field point out $n$ ranging from 2 to 16 [26, 27]. The condition $\left\|x^{+}\right\|_{2}^{2} \approx 0$ in NMR is numerically verified in [3].

Apart from the conclusion above, a remark concerning the meaning of property $(c)$ must be done: It predicts reduction in sensitivity of prescribed eigenvalues when extracted from projected companion matrices related to polynomials with $q>1$. This will be illustrated numerically later.

The following theorem states that the conditioning of the eigenvalue problem associated with $C_{A}(m, q)$ improves the conditioning of the eigenvalue problem associated with matrix $C_{A}(m, 1)$.

Theorem 4.3. Set $\breve{G}_{m}=W_{m} W_{m}^{*}$. Then for each $m \geq n$, we have that

$$
\kappa\left(G_{m, q}\right) \leq \kappa\left(\breve{G}_{m}\right) .
$$

Proof. We shall prove that

$$
\lambda_{1}\left(G_{m, q}\right) \leq \lambda_{1}\left(\breve{G}_{m}\right), \quad \text { and } \quad \lambda_{n}\left(G_{m, q}\right) \geq \lambda_{n}\left(\breve{G}_{m}\right) .
$$

In fact, let $\widetilde{\mathcal{K}}_{m}$ be as in the proof of the previous theorem. Then it is clear that $\tilde{\mathcal{K}}_{m} \tilde{\mathcal{K}}_{m}^{*}=G_{m, q}$. Using this result, for all unit vector $u \in \mathbb{C}^{n}$, we have that

$$
\begin{aligned}
u^{*} \mathcal{G}_{m, q} u & =u^{*} \tilde{\mathcal{K}}_{m} \tilde{\mathcal{K}}_{m}^{*} u \\
& =u^{*} L^{(1)} \breve{\mathcal{G}}_{m} L^{(1) *} u+\cdots+u^{*} L^{(q)} \breve{\mathcal{G}}_{m} L^{(q) *} u .
\end{aligned}
$$


Let $v_{j}(j=1: q)$ be the unit vector with the same direction as $L^{(j) *} u$. Substituting $v_{j}$ in the above equation and using the Rayleigh-Ritz characterization of eigenvalues of symmetric matrices, we get

$$
\begin{aligned}
u^{*} G_{m, q} u & =v_{1}^{*} \breve{G}_{m} v_{1}\left\|L^{(j) *} u\right\|^{2}+\cdots+v_{q}^{*} \breve{G}_{m} v_{q}\left\|L^{(q) *} u\right\|^{2} \\
& \leq \lambda_{1}\left(\breve{G}_{m}\right)\left(\left\|L^{(j) *} u\right\|^{2} \cdots+\left\|L^{(q) *} u\right\|^{2}\right) .
\end{aligned}
$$

The first inequality in (53) follows on noting that $\left(\left\|L^{(j) *} u\right\|^{2} \cdots+\left\|L^{(q) *} u\right\|^{2}\right)=1$ because by assumption all rows of $L$ have 2-norm equal to one. The second inequality in (53) follows in the same way and the proof concludes.

Note that because of its definition, whenever all $z_{j}$ fall inside the unit circle, the limit of $\mathcal{G}_{m, q}$ as $m \rightarrow \infty$ is always guaranteed to exist, and the same result applies for $\breve{G}_{m}$.

Corollary 4.4. Let $\mathcal{G}_{\infty, q}$ denote the limiting value of $\mathcal{G}_{m, q}$ as $m \rightarrow \infty$. Suppose all prescribed eigenvalues $z_{j}$ of $P_{m}(z)$ fall inside the unit circle. Define

$$
\alpha=\max \left|z_{j}\right|, \beta=\min \left|z_{j}\right|, \quad \text { and } \delta=\min \left|z_{i}-z_{j}\right|, i \neq j, 1 \leq i, j \leq n .
$$

Then

$$
\kappa\left(G_{\infty, q}\right) \leq \frac{1}{2}\left(\eta+\sqrt{\eta^{2}-4}\right)
$$

where

$$
\eta=n\left[1+\frac{n-1+\prod_{j=1}^{n}\left|z_{j}\right|^{2}-\sum_{j=1}^{n}\left|z_{j}\right|^{2}}{(n-1) \delta^{2}}\right]^{(n-1) / 2} \sqrt{\frac{1-\beta^{2}}{1-\alpha^{2}}}-n+2
$$

Proof. This corollary is a consequence of Theorem 4.3 and Corollary 9 in Bazán [5].

Example: conditioning of Mini-Mast eigenvalues. To confirm the theoretical predictions of Theorem 4.2 we have computed the condition numbers $\kappa_{q}\left(z_{j}\right)$ of the eigenvalues associated with the Mini-Mast model described in the previous section. The goal is to verify that severe reduction in sensitivity is possible when extracting the $z_{j}$ 's from projected companion matrices related to polynomials with $q>1$. Results corresponding to $q$ ranging from 1 to 4 and some values of $m$ are displayed in Table 2. Reduction in sensitivity is apparent from this table. 


\begin{tabular}{c|c|c|c|c|c|c}
\hline Mode & $\kappa_{1}\left(z_{j}\right)$ & $\kappa_{1}\left(z_{j}\right)$ & $\kappa_{2}\left(z_{j}\right)$ & $\kappa_{2}\left(z_{j}\right)$ & $\kappa_{3}\left(z_{j}\right)$ & $\kappa_{4}\left(z_{j}\right)$ \\
$j$ & $m=10$ & $m=20$ & $m=10$ & $m=20$ & $m=10$ & $m=10$ \\
\hline 1 & $0.00017 \times 10^{7}$ & $0.00130 \times 10^{3}$ & 1.77339 & 1.00929 & 1.19281 & 1.12292 \\
2 & $0.00127 \times 10^{7}$ & $0.02310 \times 10^{3}$ & 2.30402 & 1.50635 & 1.19617 & 1.04022 \\
3 & $0.00136 \times 10^{7}$ & $0.02311 \times 10^{3}$ & 1.64077 & 1.49986 & 1.13957 & 1.08176 \\
4 & $3.10889 \times 10^{7}$ & $4.75131 \times 10^{3}$ & 7.56466 & 3.86546 & 1.75153 & 1.19145 \\
5 & $3.11084 \times 10^{7}$ & $4.75306 \times 10^{3}$ & 8.07200 & 3.86827 & 1.37293 & 1.29589 \\
\hline
\end{tabular}

Table 2 - Condition numbers of prescribed eigenvalues $z_{j}$.

\subsection{An application to linear system theory}

We shall show that the Corollary 4.4 can be applied to estimating the 2-norm condition number of controllable Gramians in linear system theory. Consider a dynamical discrete linear system $S$ described by the state equations

$$
\begin{aligned}
x_{k+1} & =A x_{k}+B u_{k} \\
y_{k} & =C x_{k}
\end{aligned}
$$

where $A \in \mathbb{R}^{n \times n}, B \in \mathbb{R}^{n \times q}$, and $C \in \mathbb{R}^{q \times n}$. Assume $\lambda_{i}(A) \neq \lambda_{j}(A)$, for $i \neq j$, and $\left|\lambda_{i}(A)\right|<1(i=1: n)$. Assume also that the system is controllable, i.e., the extended controllable matrix $C_{m}^{q}$ defined by

$$
C_{m}^{q}=\left[\begin{array}{llll}
B & A B & A^{2} B \cdots A^{m-1} B
\end{array}\right], m \geq n, q \geq 1
$$

satisfies $\operatorname{rank}\left(C_{m}^{q}\right)=n$. Then the controllable Gramian of the system $S$, defined as [9]

$$
\mathcal{Q}=\sum_{j=0}^{\infty} B A^{j} A^{j *} B^{*},
$$

is guaranteed to be symmetric and positive definite, and its eigenvalues are known to concentrate information that plays a crucial role when solving system identification and model order reduction problems. It turns out that if the system eigenvalues $\lambda_{j}(A)$ are distinct, a change of basis of the state vector $\widehat{x}_{k}=T^{-1} x_{k}$ with $T$ a matrix of right eigenvectors of $A$, will transform the state space representation (54) to another one in diagonal form. When this is done, $C_{m}^{q}$ reduces to a matrix like the block Krylov matrix $\mathcal{K}_{m}$ and the controllable Gramian $\mathcal{Q}$ reduces to one like $\mathcal{G}_{\infty, q}$. This shows that the estimate for $\kappa\left(G_{\infty, q}\right)$ of the Corollary 4.4 applies to estimating the 2-norm condition number of the Gramian $\mathcal{Q}$. 


\section{Conclusions}

Based on the fact that prescribed eigenvalues of predictor polynomials can be regarded as eigenvalues of projected block companion matrices, an eigenvalue sensitivity analysis was performed. As a result, simple estimates of measures of eigenvalue sensitivity in the form of informative upper bounds were derived. In particular, under the assumption that $n$ is small, it was proved that prescribed eigenvalues near the unit circle can be relatively insensitive to noise provided the polynomial degree is large enough. The effect of the dimension of the coefficients on the sensitivity was also analyzed and it was concluded that prescribed eigenvalues of predictor polynomials can be less sensitive to noise when regarded as eigenvalues of projected companion matrices related to matrix polynomials with coefficients of order $q>1$ than when regarded as eigenvalues of projected companion matrices related to scalar polynomials. The theory was numerically illustrated using a matrix polynomial with clustered eigenvalues arising from the modal analysis field. The results are of interest in system analysis where estimates for the 2-norm condition number of controllability Gramians of multiinput multi-output discrete dynamical systems play a crucial role.

The author is aware that further research is desirable for the case where the prescribed eigenvalues are almost defective: the bounds in property (d) of Thm. 4.2 can be pessimistic in this case as the ratio $D / \delta_{j}$ is no longer small, but as illustrated in Table 2, the conditioning itself remains excellent. Furthermore, an analysis for the case where the prescribed eigenvalues are defective is needed. This challenging development is the subject of future research.

Acknowledgments. The author wishes to thank I.S. Duff and members of the ALGO Team at CERFACS for providing a cordial environment. Special thanks go to S. Gratton for suggestions that have improved the presentation of the paper. Thanks also go to the referees for their suggestions and constructive criticism. The author is particularly grateful to one referee for an important observation concerning inequality (51).

\section{REFERENCES}

[1] R.J. Allemang and D.L. Brown, A unified matrix polynomial approach to modal parameter identification, Journal of Sound and Vibration 211 (3) (1998), 323-333. 
[2] F.S.V. Bazán, Error analysis of signal zeros: a projected companion matrix approach, Linear Algebra Appl., 369 (2003), 153-167.

[3] F.S.V. Bazán, CGLS-GCV: a hybrid algorithm for solving low-rank-deficient problems. Appl. Num. Math., 47 (2003), 91-108.

[4] F.S.V. Bazán and C.A. Bavastri, An optimized Pseudo inverse algorithm (OPIA) for multiinput multi-output modal parameter identification, Mechanical Systems and Signal Processing, 10 (1996), 365-380.

[5] F.S.V. Bazán, Conditioning of Rectangular Vandermonde Matrices with nodes in the Unit Disk, SIAM J. Matrix Analysis and Applications, 21 (2) (2000), 679-693.

[6] F.S.V. Bazán and Ph.L. Toint, Error analysis of signal zeros from a related companion matrix eigenvalue problem, Applied Mathematics Letters, 14 (2001), 859-866.

[7] F.S.V. Bazán and Ph.L. Toint, Singular value analysis of predictor matrices, Mechanical Systems and Signal Processing, 15 (4) (2001), 667-683.

[8] F. Chaitin-Chatelin and V. Frayssé, Lectures on Finite Precision Computations. SIAM, Philadelphia (1996).

[9] Chi-T. Chen, Linear System theory and Design, Third Edition, Oxford University Press, New York (1999).

[10] L. Elsner and M.C. Paardekooper, On measures of nonnormality of matrices, Linear Algebra Appl., 92 (1987), 107-124.

[11] G.H. Golub and C.F. Van Loan, Matrix Computations, The Johns Hopkins University Press, Baltimore (1996).

[12] I. Gohberg, P. Lancaster and L. Rodman, Matrix Polynomials, Academic Press, New York (1982).

[13] P. Henrici, Bounds for iterates, inverses, spectral variation and field of values of nonnormal matrices, Numer. Math. 4 (1962), 24-40.

[14] D.J. Higham and N.J. Higham, Structured Backward error and condition of generalized eigenvalue problems, SIAM J. Matrix Anal. Appl. 20 (2) (1998), 493-512.

[15] N.J. Higham and F. Tisseur, Bounds for eigenvalues of Matrix Polynomials, Linear Algebra and Its Applications, 358 (2003), 5-22.

[16] R. Horn and Ch.R. Johnson, Matrix Analysis, Cambridge University Press (1999).

[17] F. Li and R.J. Vaccaro, Unified analysis for DOA estimation algorithms in array signal processing, Signal Processing, 25 (1991), 147-169.

[18] J.-Lew, J.-N. Juang and R.W. Longman, Comparison of several system identification methods for flexible structures, Journal of Sound and Vibration 167 (3) (1993), 461-480.

[19] Jer-Jan Juang and R.S. Pappa, An Eigensystem Realization Algorithm for Modal Parameter Identification and Model Reduction, J. Guidance, Control and Dynamics, 8 (5) (1985), 620-627. 
[20] Z. Liang and D. J. Inman, Matrix Decomposition Methods in Experimental Modal Analysis, Journal of Vibrations and Acoustics, Vol 112 (1990), 410-413, July 1990.

[21] B.D. Rao, Perturbation Analysis of an SVD-Based Linear Prediction Methods for Estimating the Frequencies of Multiple Sinusoides, IEEE Trans. Acoust. Speech and Signal Processing ASSP 36 (7) (1988).

[22] A. Sinap and W. Van Assche, Orthogonal matrix polynomials and applications, Journal of Computational and Applied Mathematics, 66 (1996), 27-52.

[23] R.A. Smith, The condition numbers of the matrix eigenvalue problem, Numer. Math., 10 (1967), 232-240.

[24] F. Tisseur, Backward error and condition of polynomial eigenvalue problems, Linear Algebra Appl., 309 (2000), 339-361.

[25] A. Van Der Veen, E. F. Deprettere and A. Lee Swindlehurst, Subspace-Based Signal Analysis Using Singular Value Decomposition, Proceedings of the IEEE, 81 (9) (1993), 1277-1309, September 1993.

[26] S. Van Huffel, Enhanced resolution based on minimum variance estimation and exponential modeling, Signal Processing, 33 (1993), 333-355.

[27] S. Van Huffel, H. Chen, C. Decanniere and P. Van Hecke, Algorithm for time domain NMR datta fitting based on total least squares, J. Magnetic Resonance, A 110 (1994), 1277-1309.

[28] Q.J. Yang et al., A System Theory Approach to Multi-Input Multi-Output Modal parameters Identification Methods, Mechanical Systems and Signal Processing 8 (2) (1994), 159-174.

[29] L. Zhang and H. Kanda, The Algorithm and Application of a new Multi-Input-MultiOutput Modal Parameter Identification Method, Shock and Vibration Bulletin, pp. 11-17, (1988).

[30] J.H. Wilkinson, The Algebraic Eigenvalue Problem, Oxford University Press, Oxford, UK (1965). 